\title{
August 2013 Arizona Thoracic Society Notes
}

The August Arizona Thoracic Society meeting was held on Wednesday, 8/28/2013 at Shea Hospital beginning at 6:30 PM. There were 23 in attendance representing the pulmonary, critical care, sleep, and pathology communities.

A brief discussion was held about the audio-visual aids available. It was generally agreed that our current projector is inadequate. Judd Tillinghast will inquire about using a hospital overhead projector. If that is not possible, it was agreed to purchase a new projector.

Plans for telecasting the meeting between Phoenix and Tucson continue. A trial of a link between Shea and the University in Tucson failed. Once the link is successfully established, it is hoped that the meeting can be telecasted.

There were 6 cases presented:

1. Dr. Thomas Colby, pulmonary pathologist from Mayo Clinic Arizona, presented the case of a 10 year old boy with chronic dyspnea for $>4 \mathrm{yrs}$. He had growth retardation since age 1, a skin rash since age 2 on the limbs, nail dystrophy since age 3 on hands and feet, lacrimal duct stenosis, erythematous lesions on the pinnae, phimosis, interstitial lung disease on radiography, weakly positive p-ANCA, elevated erythrocyte sedimentation rate, and hypergammaglobulinemia. He came to lung biopsy. The patient was diagnosed with dyskeratosis congenita which is a disorder of poor telomere maintenance (1). Specifically, the disease is related to one or more mutations which directly or indirectly affect the vertebrate telomerase RNA component (TERC). This patient's manifestations are fairly typical of the disease. Short telomere length was confirmed.

2. Dr. Colby presented a second case of a 14 year old boy with a history of osteosarcoma. Pulmonary nodules developed and biopsy showed metastatic osteosarcoma. He was given systemic chemotherapy but now has residual nodules that were biopsied. One of the pulmonary nodule resembled bronchoalveolar cell carcinoma. This is an apparent complication following chemotherapy in adolescent patients (2).

3. Dr. Colby presented the pathology of a patient from the Phoenix VA who underwent lung biopsy for interstitial disease. The pathology was typical for IgG4-related disease with a plasma cell rich lymphohistiocytic infiltrate in the bronchovascular sheath and histopathology showing diffusely stained positive for IgG4 plasma cells (3).

4. Dr. Suresh Uppalapu, a second year pulmonary fellow from Good Samaritan/VA, presented a 59 year old Sudanese male who was transferred to the Good Samaritan ICU in shock. His presenting complaints to the 
transferring hospital were acute mental status changes, weakness, and chills. He was intubated for hypercarbic respiratory failure. His brother related that the patient had just returned from Sudan three weeks earlier. He had a prior history of a splenectomy. He was hypothermic with a temperature of $32.3^{\circ} \mathrm{C}$ and a SpO2 of $91 \%$ on $100 \%$ FiO2 and PEEP of 8 . His Glasgow Coma Scale was 3 (lowest possible score). He had many abnormalities on laboratory evaluation, most notably a creatinine of $5.1 \mathrm{mg} / \mathrm{dL}$ and a lactic acid of 26.3 $\mathrm{mg} / \mathrm{dL}$. The peripheral smear showed malaria parasites typical of falciparum malaria (figure 1).

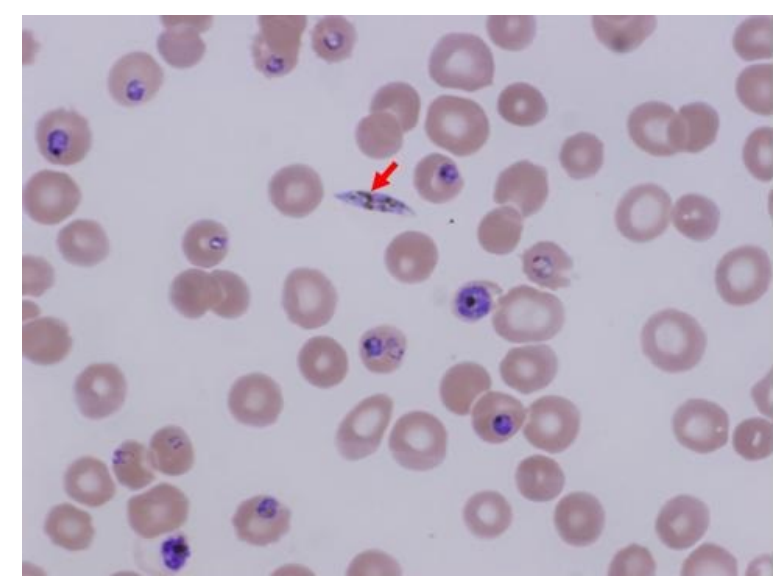

Figure 1. Peripheral smear showing a gametocyte (red arrow) and trophozoites in various stages from falciparum malaria.

He developed hemoptysis and eventually expired. A preliminary autopsy report has detected aspergillosis in the lung. Invasive aspergillosis has been reported in cases of severe falciparum malaria (4).

5. Dr. Heemesh Seth, also a second year pulmonary fellow from Good Samaritan/VA, presented a case of a 57 year old man with cirrhosis secondary to hepatitis $C$ diagnosed in 1998. He presented with a large right hydrothorax. Multiple thoracentesis were performed (Table 1).

Table 1. Summary of multiple thoracentesis.

\begin{tabular}{|c|c|c|c|c|c|c|c|c|c|}
\hline Date & Fluid & Color & Clarity & WBC & RBC\% & Segs\% & Lymphs\% & Monos\% & Eos\% \\
\hline Aug 9 & Pleural & Yellow & Hazy & 311 & 2 & 57 & 28 & 3 & 0 \\
\hline Aug 11 & Pleural & Yellow & Turbid & 15751 & 99 & 0 & 1 & 0 & 0 \\
\hline Aug 13 & Pleural & Yellow & Cloudy & 15569 & 96 & 1 & 3 & 0 & 0 \\
\hline Aug 16 & Pleural & Amber & Cloudy & 4620 & 90 & 6 & 1 & 3 & 0 \\
\hline Aug 19 & Pleural & Red & Cloudy & 2268 & 65 & 13 & 0 & 0 & 2 \\
\hline Aug 20 & Peritoneal & Yellow & Clear & 94 & 38 & 10 & 0 & 23 & 0 \\
\hline Aug 23 & Pleural & Red & Bloody & 2520 & 77 & 18 & 0 & 1 & 4 \\
\hline Aug 26 & Pleural & Red & Cloudy & 1407 & 61 & 32 & 0 & 0 & 7 \\
\hline
\end{tabular}


Blood cultures were positive for acinetobacter as was the initial culture from the thoracentesis. He was treated with cephepime. It was felt that his effusion and empyema were most likely secondary to translocation of bacteria to the pleural space from spontaneous bacterial peritonitis. A discussion ensued regarding whether to perform tube thoracostomy. Data is sparse with most literature not favoring a chest tube (5). However, in this patient's case a chest tube was eventually inserted when he failed to improve. It drained about 2 liters of fluid but the drainage then became minimal and the tube was removed. The patient developed hepato-renal syndrome but was felt not to be a liver transplant candidate. He was transferred to hospice.

6. Dr. Seth also presented a second case of a 66 year old Hispanic man who presented with a large left pleural effusion. He had a past medical history of systemic lupus erythematosis (SLE) with possible rheumatoid arthritis and was being treated with adalimumab, methotrexate, and prednisone. A thoracentesis was done and 2 liters of clear amber fluid was removed. Although be developed fever to $102^{\circ} \mathrm{F}$ he felt much better the next morning and was discharged. However, his coccidioidomycosis serologies were positive for both IgG and IgM and his complement fixation test were positive at 1:4. Pleural fluid cytology was positive for LE cells. He was continued on prednisone and treated with fluconazole. A discussion developed of whether the effusion was secondary to SLE, coccidioidomycosis, or both. No one knew data but it was felt that it was most prudent to continue the present course while following the patient and awaiting cultures.

There being no further business the meeting was adjourned at about 8:15 PM. The next meeting is scheduled for Wednesday, September 25, 6:30 PM in Phoenix at Scottsdale Shea Hospital.

Richard A. Robbins, M.D.

\section{References}

1. Dokal I. Dyskeratosis congenita in all its forms. $\mathrm{Br} \mathrm{J}$ Haematol. 2000;110(4):768-79. [CrossRef] [PubMed]

2. Travis WD, Linnoila RI, Horowitz M, Becker RL Jr, Pass H, Ozols R, Gazdar A. Pulmonary nodules resembling bronchioloalveolar carcinoma in adolescent cancer patients. Mod Pathol. 1988;1(5):372-7. [PubMed]

3. Hurley JR, Leslie KO. IgG4-Related systemic disease of the pancreas with involvement of the lung: a case report and literature review. Southwest J Pulm Crit Care. 2013;7(2):117-130. [CrossRef]

4. Hocqueloux L, Bruneel F, Pages CL, Vachon F. Fatal invasive aspergillosis complicating severe Plasmodium falciparum malaria. Clin Infect Dis. 2000;30(6):940-2. [CrossRef] [PubMed]

5. Alonso JC. Pleural effusion in liver disease. Semin Respir Crit Care Med. 2010;31(6):698-705. [CrossRef] [PubMed] 